\title{
Establishment of laboratory quality control indicators for NAT on blood donors' samples
}

\author{
Ziyi He*, Shaobin Chen, Lin Yu, Qing Wang, Jiaoli Zou \\ Department of Transfusion Research, Dongguan Blood Center, Dongguan, Guangdong, 523930, China
}

\begin{abstract}
In order to establish and screen nucleic acid test (NAT) quality control indicators for blood donor samples, $\mathrm{C}_{\mathrm{T}}$ value of each test item were detected by NAT, internal and external quality controls, the number of unsuccessful mixed samples, the split positive rate, the rate of detection efficiency, and the equipment failure rate were collected. The "instant method" is used to establish the Levey-Jennings quality control chart, the mixed test positive rate, the rate of unqualified sample. Possion distribution is used to establish the quality control chart, and it is easily to operate and monitor NAT effectively. The results displayed that quality control charts were established, including unqualified sample Possion distribution quality control chart, the rate of equipment failure quality control chart, the failure rate of reagent batch and inefficient quality control chart, test result positive of Possion distribution quality control chart, quality control charts of NAT positive Possion distribution probability, the rate of split positive quality control charts, quality control charts of unsuccessful mixed samples, quality control chart of reagents effective rate, internal quality control chart, correctness, and NAT qualitative quality control chart. The study established quality control indicators throughout the whole NAT process, which were able to effectively improve NAT quality control and the efficacy of laboratory management.
\end{abstract}

Keywords: NAT, invalid results, quality control indicator, Possion distribution, quality control chart

\section{INTRODUCTION}

Since 2010, in China nucleic acid testing (NAT) has been performed in 15 blood centers. In 2013, the Chinese National Health Institute issued an implementation plan for promoting NAT(2013-2015), which required NAT to be implemented in all blood centers. Since then, some blood centers have implemented NAT, and have accumulated valuable experience in the development of testing methods, laboratory renovation, instrument performance verification, software upgrades, the testing process and risk assessment ${ }^{[1-3]}$. However, many blood centers still use external quality controls for $\mathrm{HBV} / \mathrm{HCV} / \mathrm{HIV}$ standard products rather than internal quality controls or mix three quality controls into one for each test to ensure its concentration of 2-5 times LoD (limit of detectable) as the quality control indicator ${ }^{[4]}$. For best effect, these should be combined with internal quality controls to compare the differences between different NAT systems in the same laboratory ${ }^{[5]}$. In 2017, National Health and Family Planning Commission's Clinical Test Center issued the clinical laboratory quality indicators of China, which includes 28 important quality indicators: i.e. 12 indicators in the pre-test process; 8 in the test phase; 5 in post-test; as well as 3 in supporting indi-

*Correspondence to: Ziyi He, MD, Department of Transfusion Research, Dongguan Blood Center, 19 Ningjing Road, Humen Town, Dongguan, Guangdong, 523006, China. TEL: 0086-769-85152673, E-mail: zyhe_8@163.com.

Conflict of interests: The authors declared no conflict of interests. 
cators. Comprehensive quality managements including personnel training, reagents, instrument, samples, and results are also released. As yet, laboratory quality control indicators have not fully been established in blood centers, therefore it needs to establish some quality control indicators which can monitor the whole process ${ }^{[6]}$. The purpose of establishing these quality indicators is for the statistical analysis of laboratory data retrospectively, pollution evaluation, detection of any system errors, and normalization of operation.

\section{MATERIALS AND METHODS}

\section{NAT kit and instruments}

Roche Cobas TaqScreen MPX v2.0 HIV/HCV/ HBV(Roche), Hamilton STAR (MICROLAB STAR IVD, Hamilton, Switzerland), Automatic NAT system (Cobas s201, Roche). All reagents were used within their validity period, and all instruments were calibrated and under normal conditions.

\section{Internal quality control}

HIV RNA standard (Lot: 201309001, concentration: 1,000 IU/mL), HCV RNA standard (Lot: 201309001, concentration: 1,000 IU/mL), HBV DNA standard (Lot: 201309001, concentration: $200 \mathrm{IU} / \mathrm{mL}$ ), came from Bao Ruiyuan Biotechnology Co., LTD (Beijing, China). Quality control product preparation: according to the HCV RNA, HBV DNA and HIV RNA provided by Cobas TaqScreen MPX2.0 detection reagent specification, the initial concentration of the mixed quality control is about 2-5 times of LoD. HCV RNA, HBV DNA, HIV RNA and negative plasma were mixed at a ratio of 1:1:5:23. The theoretical HCV RNA, HBV DNA and HIV RNA concentrations were $33.33 \mathrm{IU} /$ $\mathrm{mL}, 6.67 \mathrm{IU} / \mathrm{mL}$ and $166.67 \mathrm{IU} / \mathrm{mL}$, respectively. Each $4.5 \mathrm{~mL}$ new quality product was placed into sterilized tubes respectively, then separated into 7 tubes, and stored at $4^{\circ} \mathrm{C}$.

\section{Data collection}

At least 30 original NAT records, taken from our laboratory in Mar. 2018 were collected randomly, including $\mathrm{C}_{\mathrm{T}}$ values of external quality control IC, HIV, $\mathrm{HBV}, \mathrm{HCV}$, internal quality control MPC(mix positive control) IC, negative control IC, MPC HIV, MPC HBV, MPC HCV, HIV-2, HIV-2 IC, HIV-10 and HIV-10 IC. The test failure rate was collected from 2016 to 2017 , and the instrument failure rate was collected in each year from 2012 to 2017, respectively. The effective utilization rate of reagent was collected monthly from 2016 to 2017 , and the unsuccessful rate of mixed samples were collected from 2015 to 2017. The above data were all tabulated using Microsoft Excel.

\section{NAT procedures}

NAT testing followed standard blood center operating procedures: 1 pool of negative control, 3 pools of positive control (MPC, HIV-10, and HIV-2), 1 pool of external quality control, and other 20 samples pools were used for each batch of testing .

\section{Establishment of $\mathbf{C}_{\mathrm{T}}$ value quality control chart}

The following $\mathrm{Ct}$ value quality control chart was established, seeing reference ${ }^{[7]}$. The first three points are used to calculate the average, and the CV control limit is $20 \%$. Any values of more than 2 standard deviations (SD) are taken out. Once 20 units of data were filled, the quality control chart would be plotted as mean value and SD. The Ct value is detected by NAT, with the mean $\pm 2 \mathrm{SD}$ as the upper warning line, while the out of control limit is the mean \pm 3 SD. One mixed QC for each batch of routine samples is taken as positive control every day, the $\mathrm{Ct}$ value of $\mathrm{HCV}$ RNA, HBV DNA and HIV RNA is recorded for $20 \mathrm{~d}$ continuously, the mean, SD and coefficient of variation are calculated and a Levey-Jennings quality control chart is plotted.

\section{Establishment of the control limit for other in- dicators}

The control limit for the normal distribution of the counting data is based on the statistically significant limit of 0.05 , and the single tail only one out of control limit. For small probability events, the probability value is calculated based on Poisson distribution, and the out of control limit is $P<0.05$.

According to the specification of Roche NAT, the maximum fault tolerance rate (out of control limit) of the failed pool is $1.4 \%$ and the maximum fault tolerance rate (out of control limit) of rack failure is $4.7 \%$.

\section{Selection of quality control rules}

The rules of quality control were selecated according to the rules of clinical significance. Westgard quality control standards can be set as: $1_{3 s}, 2_{2 s}, R 4_{s}, 4_{1 s}$, $10 \mathrm{x}, 7 \mathrm{~T}$, etc.

\section{RESULTS}

\section{Qualitative control chart of NAT}

NAT is a qualitative method, with negative or positive test results and no quantitative data. Therefore, 
the quality control chart cannot be drawn according to the quantitative detection method. For qualitative tests such as NAT, positive quality controls with a concentration range of $2-5$ times the LoD reading and nega-
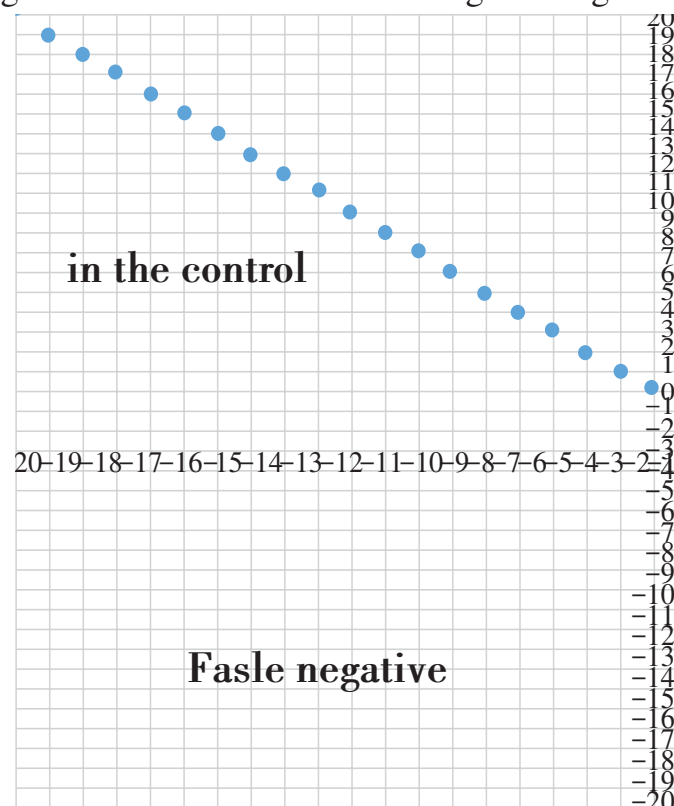

tive quality control can be selected. Below are quality control charts based on the qualitative detection method (Fig.1). If a false positive or false negative reaction occurs, it is deemed out of control.

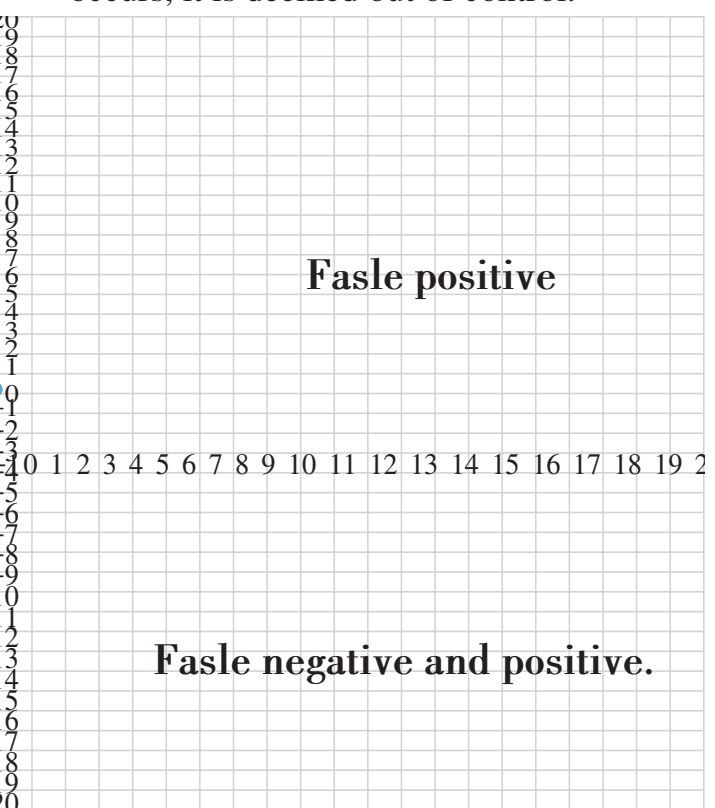

(Note: positive vertical value means positive quality control product testing, and negative horizontal value means negative quality control product testing).

Fig. 1 Quality control charts of qualitative detection

\section{Quality control chart of internal quality con- trol (MPC of HIV/HBV/HCV/IC $\mathrm{C}_{\mathrm{T}}$ values)}

For Ct values of HIV, HBV, HCV and IC for the internal quality control products, the "instant method" was used to establish the internal quality control chart (Fig.2), and 20 points were selected in the controllable range. The lower the $\mathrm{Ct}$ value, the higher the target concentration, while the lower the warning line and the lower the out of control line and the higher the probability of false positive detection. The internal quality control chart based on $\mathrm{Ct}$ value can dynamically monitor the stability of the test, performance of reagents or the presence of inhibitors.

\section{Quality control chart of external quality con- trol (HIV/HBV/HCV/IC C $\mathrm{C}_{\mathrm{T}}$ values)}

The Ct values of IC, HIV, HBV and HCV for the external quality control were respectively established by "instant method" (Fig.3).

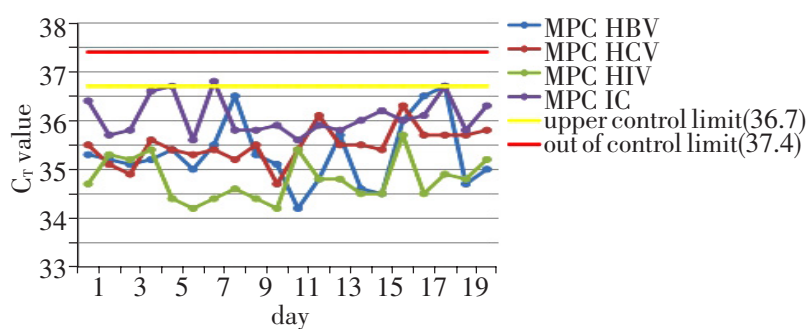

Fig. 2 Internal quality control chart of HIV, HBV, HCV and IC Ct values

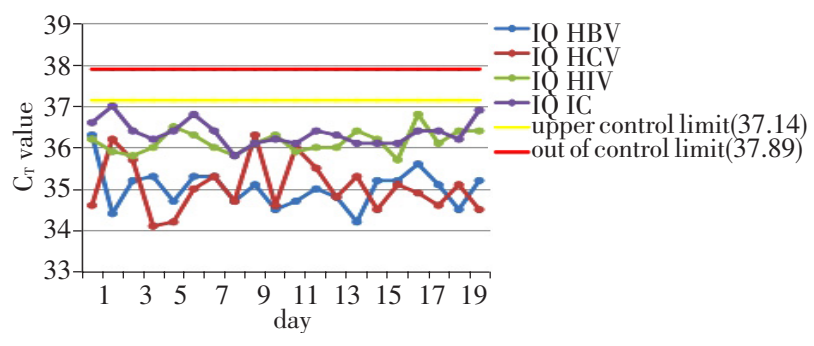

Fig. 3 Quality control chart of external quality control HIV/HBV/HCV/IC $\mathrm{C}_{\mathrm{T}}$ values

\section{Quality control chart for the Possion distri- bution of unqualified samples}

Totally 273,364 samples were received from Jan $1^{\text {st }}, 2015$ to May $31^{\text {st }}, 2018$. There were 59 unqualified sample cases, at a rate of $1 / 4,633$. Statistical probabilities for the unqualified samples were calculated according to the Possion distribution every month, with 0.05 as the out of control limit. The Poisson distribution quality control chart for unqualified samples from 2016 was shown below (Fig.4). As can be seen from Fig.4, a total of 8 cases of unqualified samples occurred in June, so the probability of disqualification was lower than the control limit. The quantity of unqualified samples is out of control limit, therefore for this month the distribution of disqualification conclusion was insufficient quantity and hemolysis. 


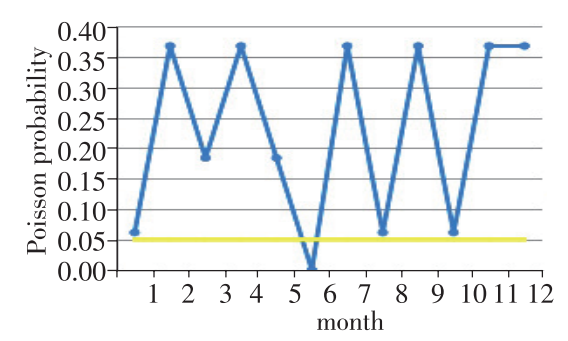

Fig. 4 Quality control chart for the Possion distribution of unqualified samples

\section{The quality control chart for unsuccessful mixed samples}

The unsuccessful mixed sample rate was calculated according to the unsuccessful mixed samples tested in 2016. The upper limit was the average rate of unsuccessful mixed samples $\pm 2 \mathrm{SD}$. The quality control chart was drawn according to the monthly rate of unsuccessful mixed samples in 2016 years (Fig.5). The chart shows that no unsuccessful mixed samples out of control samples occurred in 2016.

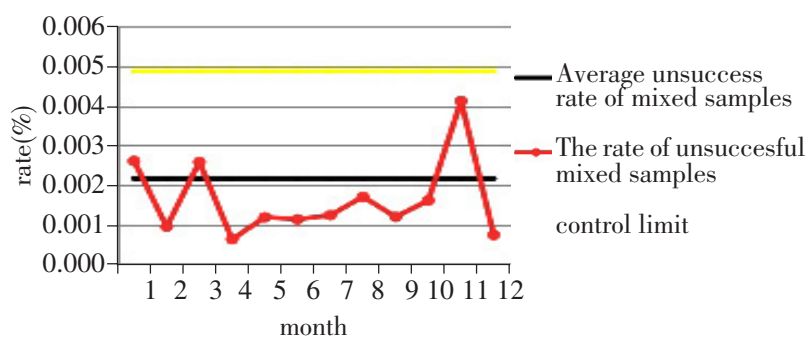

Fig. 5 The quality control chart for unsuccessful mixed samples

\section{Over 72 h NAT rate}

The number of samples whose NAT results were not issued for more than $72 \mathrm{~h}$ every month from Jan to Dec 2017 were recorded. These data were compared with the number of samples received for the month, so the detection rate of over $72 \mathrm{~h}$ every month was calculated, and the quality control chart of over $72 \mathrm{~h}$ NAT test was drawn. NAT for over $72 \mathrm{~h}$ was available every month in 2017 (Fig.6). The possible causes for the NAT failure over $72 \mathrm{~h}$ were unsuccessful sample mixing, instrument failure, or difficult blood group retesting.

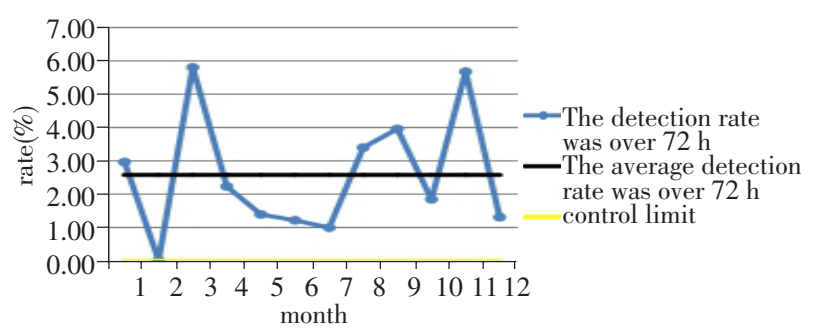

Fig. 6 Quality control chart of over 72 h NAT

\section{Quality control chart of test failure rate}

According to the Roche s201s' instruction, the maximum probability of an invalid test for a single pool is $1.4 \%$. The number of pools which resulted in an invalid test were calculated every month from 2016 to 2017. With $1.4 \%$ as the out of control limit, the quality control chart of test failure rate was drawn (Fig.7). The results showed 9 instances over two years with an invalid test rate, which were over $1.4 \%$ out of control.

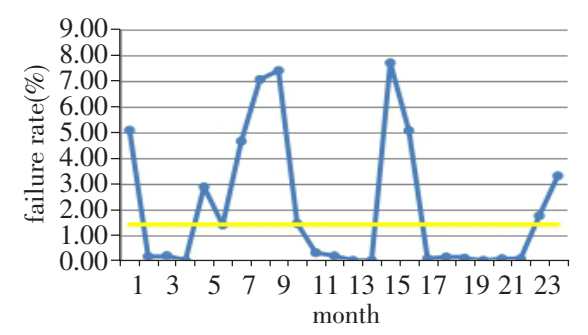

Fig. 7 The quality control chart of test failure rate

\section{Quality control chart of instrument failure rate}

The quality control chart of instrument failure rate was drawn according to the probability of instrument failure every year, with an out of control limit of 0.05 (Fig.8). The chart showed two NAT instruments were out of control, while the 888 NAT instrument has been under control for four years and is relatively stable. This showed that different instruments have different failure rates which are independent to operator proficiency and routine maintenance.

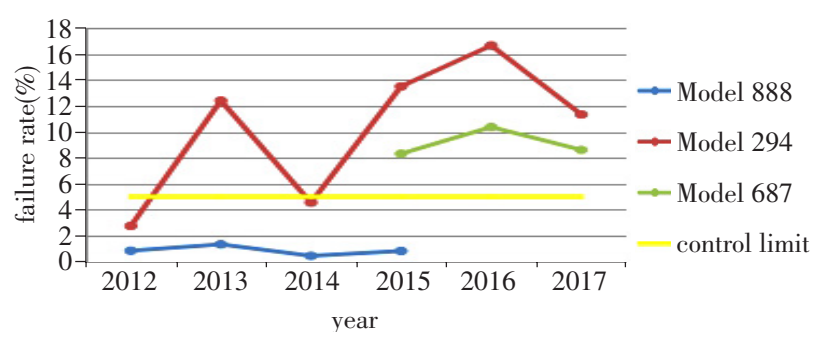

Fig. 8 Quality control chart of instrument failure rate

\section{Quality control chart of reagent batch num- ber and inefficiency}

The different batches of reagents and inefficiencies used in the laboratory were compared and the quality control chart of reagent batch and inefficiencies was produced (Fig.9). The total invalid maximum tolerance was set at $4.7 \%$ and the single pool invalid maximum fault tolerance (control limit) was set as $1.4 \%$. Of the eleven batches of reagents used in our laboratory in 2017 , two batches of reagents exceeded the control limit and therefore failed. HCV reaction was found to have occurred in the negative control, perhaps due 
to the presence of an inhibiting substance, laboratory pollution, or reagent instability. This is a valuable reference to quality control in a laboratory environment, including reagents, the transport, and sample contamination.

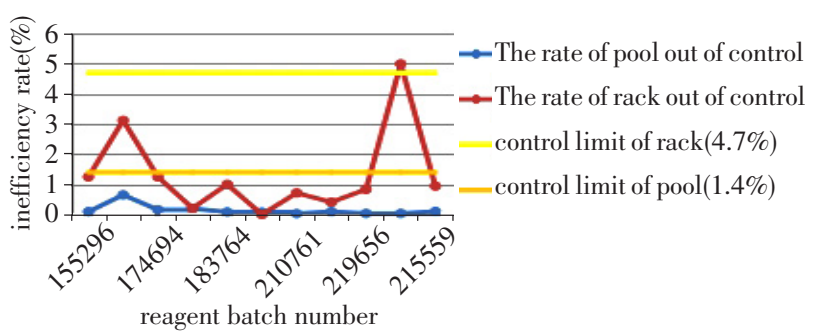

Fig. 9 Quality control chart of reagent batch number and inefficiency

\section{Quality control chart of NAT positive Poisson distribution}

The average positive rate of NAT from Oct. 2014 to Dec. $31^{\text {st }}, 2017$ was taken as $\lambda$ value. The Poisson distribution probability of positive NAT was calculated every month, with a $P$ value of 0.05 as the quality control limit. The lower probability of the uncontrolled control means that there may have been contamination, inversely; it may have been that the laboratory had a nucleic acid amplification inhibitor. A quality control chart of Poisson distribution was drawn, based on the positive test results from our laboratory every month in 2016 (Fig.10). This showed the situation was out of control for 4 months, so the cause should be investigated from the aspect of contamination and presence of inhibitors.

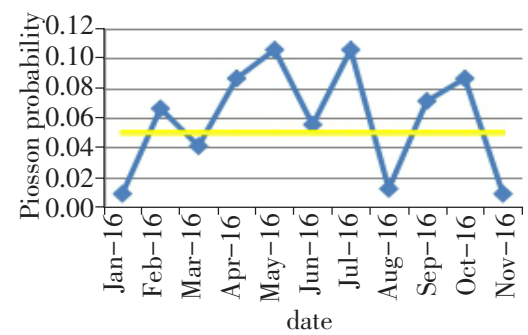

Fig. 10 Quality control chart of NAT positive Poisson distribution

\section{Quality control chart of monthly split positive rate}

The average split positive rate was calculated for the total number of mixed positive tests and split positive tests every month from 2015 to 2017, with the average split positive rate set as mean+2SD for the control limit, and the quality control chart of monthly split positive rate in 2016 was drawn (Fig.11). The possible reasons for the split positive rate over the upper control limit indicated laboratory pollution, while the possible reasons for the lower control limit were either an inhibitor in the laboratory or improper sample preservation before testing.

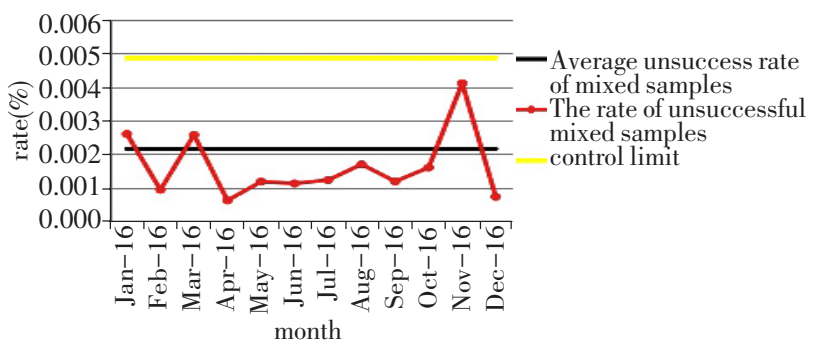

Fig. 11 Quality control chart of monthly split positive rate

\section{Quality control chart for effective use of reagents}

The formula for the effective use of reagent is NAT sample number/reagent dosage. The average rate of reagent effective use was calculated over three years from 2015 to 2017, the lower limit was the average rate $-2 \mathrm{SD}$, and the quality control chart was drawn (Fig.12). As can be seen, there was one reagent effective use rate in our laboratory out of control in 2017, as multiple NAT tests were ineffective that month, requiring retests due to possible issues with the detection system.

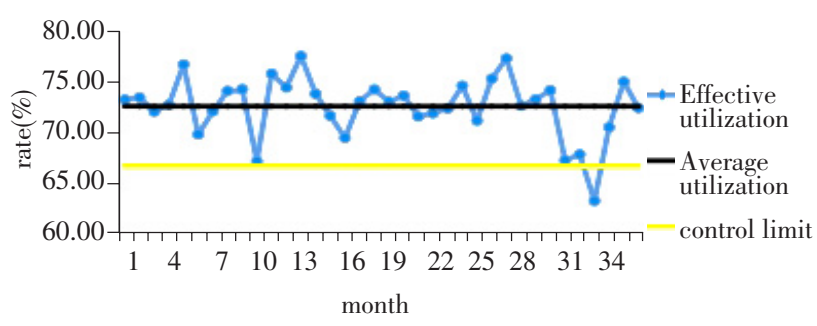

Fig.12 Quality control chart of effective utilization of reagents

\section{DISCUSSION}

Internal quality control is important to measure the precision of daily tests in laboratories ${ }^{[8]}$. In qualitative detection, this can not only monitor the daytime precision, but can also be an important indicator for the sensitivity of the detection system ${ }^{[5]}$. The implementation of quality control through the establishment of quality indicators is able to show the degree of a set of inherent characteristics in order to meet the requirements of measurement ${ }^{[9]}$. This can not only evaluate each key step in the whole process, but can also inspect processes, including safety and environment, instrumentation, the effectiveness of the personnel, and documents of quality control ${ }^{[10]}$. In Feb. 2017, the clinical examination center for the National Health and Family Planning Commission of China proposed 
28 quality indicators for clinical test, among which 12 were for pre-test, 8 in test, 5 in post-test and 3 supporting indicators ${ }^{[11]}$. However, the detection is limited by the 2015 edition of the Blood Station Technical Operation Procedure in blood centers. Except for ALT, all tests were qualitative detection, but as yet no corresponding quality indicators for internal quality control are issued. Especially, after NAT was implemented, the question of how to perform quality control of NAT and establish effective quality indicators has become a primary problem. Although NAT as a qualitative test, the $\mathrm{Ct}$ value is also the basis for estimating whether the result is positive or negative, therefore, some laboratories used the $\mathrm{Ct}$ value as the internal quality indicator according to the quantitative detection method, and established a Levey-Jennings quality control chart. This study also used $14 \mathrm{Ct}$ values generated in the process of NAT for different items, resulting in 14 different quality control charts. However, the quality control chart cannot achieve the goal of internal quality control. When IC for sample $\mathrm{Ct}$ value is used to establish a quality control chart, although samples are positive, the IC may be negative, and this means IC Ct value is not exist, but sample result could show as effective. However, the internal quality control may deem the same sample out of control, due to the standards for internal controls being more sensitive. Routine practice in all blood centers used external quality control products to dilute $\mathrm{HBV} / \mathrm{HCV} /$ HIV concentration to $2-5$ times LoD of the NAT system, and the upper and lower control limit were set according to the method of mean $\pm 3 \mathrm{SD}$. The smaller the $\mathrm{Ct}$ value, the higher the concentration of the target detector, so the setting of the minimum out of control limit is unnecessary. Setting the maximum out of control limit to control false positive, even if the higher $\mathrm{Ct}$ value exceeds the upper out of control limit, cannot be interpreted as out of control. A high $\mathrm{Ct}$ value is reactive and controlled qualitatively. In most cases, the coefficient of variation for the $\mathrm{Ct}$ value is small, or the concentration marked by the external quality control product is lower than the actual concentration, which is used for NAT after dilution. The $\mathrm{Ct}$ value fluctuates little with the number of detection or time, so it is difficult to achieve the purpose of quality control.

To enhance and improve the quality of NAT, the quantitative evaluation of quality management is achieved by monitoring and recording the key control points before, during and after the test. In this way, problems in the laboratory can be found in time. According to each clinical situation, the quality indicators including the rate of unqualified samples, the sample positive rate, the rate of equipment failure, and the inefficient rate of reagent were recoded, the results were analyzed and a quality control chart was plotted, in order to monitor and discover unqualified, invalid results, such as out of control data or equipment failure. In this way, NAT samples could be important to ensure with accuracy, even a long time after blood sample collection, hemolysis, bacterial infection, dissociation of the gel in plasma ${ }^{[12]}$, and insufficient centrifugation could affect the NAT results. In this paper, two indicators reflecting the quality of blood samples, namely, the occurrence rate of unqualified samples and the unsuccessful mixed samples rate, were selected for sample management quality control, which could response the situation of out of control limit on sample management and suggest to strengthen sample management and guarantee quality. We should pay attention to blood sample collection and have corresponding quality control indicators. At the same time, we examined quality control on the failure rate of different NAT systems, and found that the occurrence of failure rate of different NAT systems is irrelevant to operator, environment, reagents or other factors, but is closely related to the performance of the system. Through the analysis of the inefficiencies of different reagent batches, it was found that there was a close relationship between the quality of reagent and the environment pollution. We conducted quality analyses on the positive number or positive rate after testing, and found that the fluctuation was relatively large, and the data characteristics were in line with Poisson distribution. With the establishment of quality control charts, the possibility of laboratory pollution and the safety of blood donors can be further safeguarded. The quality control chart of the split positive rate after the mixed sample positive NAT can also monitor whether there is contamination in the laboratory or deficiencies in the quality of sample preservation. The quality control chart of the effective use rate of NAT reagents can be used to assist cost-benefit analysis in the laboratory, which is helpful to improve the effective use rate of reagents. To ensure the accuracy of the DNA laboratory, we used the unqualified samples Poisson distribution quality control chart, unsuccessful mixed samples, quality control chart for test failure rate, the number of testing positive for Poisson distribution chart, and resolution rate of monthly quality control chart as conventional NAT laboratory quality control indicators. The rest of the quality indicators were acted as supplement to monitor NAT laboratory quality, in order to ensure that NAT met the needs of blood safety.

Each laboratory should strive to perform adequate internal quality controls, to ensure that the whole processes from sample collection, DNA extraction and 
amplification to detection are effective, and to monitor the key control points. Only complete quality control indicators can realize reasonable, controllable, orderly, accurate and timely results. The major benefits of using this system are as follows, each laboratory can select and develop quality indicators according to its actual conditions, and adjust the detection frequency of each monitoring quality indicator according to specific conditions to ensure the key points, so as to guarantee the internal quality control of NAT.

\section{References}

[1] He ZY, Chen SB, Yu L, et al. Analysis and application of NAT in Dongguan blood center more than 10 years. Asia-Pacific Journal of Blood Types and Genes, 2018, 2(2):97-102.

[2] He ZY, Chen QK, Chen SB, et al. The residual risk of the blood transmitted virus in four detection models. Chin $J$ Blood Transfusion(in Chinese), 2018, 31(2):140-4.

[3] Chen SB, He ZY, Chen QK, et al.Performance verification of Roche Cobas s201 nucleic acid test system in Dongguan Blood Center. Asia-Pacific Journal of Blood Types and Genes, 2018, 2(3):171-5.

[4] Chen SB, He ZY, Yu L, et al. Application analysis of multi-labeled internal quality control products in blood nucleic acid screening. Chin J Blood Transfusion(in Chinese), 2016, 29 (4): 423-6.

[5] Wang LN. Laboratory capacity building of blood station. Chin J Blood Transfusion(in Chinese), 2008, 31 (2): 99-
100

[6] Wang R, Wu SH, Gao F, et al. Establishment and application of blood station laboratory quality monitoring indictors system. Chin J Blood Transfusion(in Chinese), 2011, 24 (8): 713-5.

[7] Wang K, Wu ZHD. Application of Excel in "instant method" indoor quality control. Chin J Blood Transfusion(in Chinese), 2008, 31 (2): 195-7.

[8] Zheng HJ. Indoor whole-process quality control for laboratory immunology tests of blood stations at all levels must be done. Chin J Blood Transfusion(in Chinese), 2002, 15 (5):299-300.

[9] ISO. ISO 15189, Medical laboratories-requirements for quality and competence. 3rd ed. Geneva: International Organization for Standardization, 2012.

[10] Wang ZHG, Fei Y, Kang FF, et al. Content and interpretation of 15 medical quality control indicators for clinical laboratory specialty (2015 edition) issued by the national health and family planning commission. Chin J Blood Transfusion(in Chinese), 2015, 38(11):777-81.

[11] Ye YY, Wang W, Zhao HJ, et al. Progress in establishment and application of clinical test quality indexes in China. Chin J of Clin Lab Management (in Chinese), 2016, 4 (4):227-9.

[12] Chen SB, He ZY, Chen QK. Analysis of causes of nucleic acid detection failure in blood donor specimens due to "clotting". Chin J Blood Transfusion(in Chinese), 2008, 31 (2): 192-4.

(Received 23 November 2018, Revised 22 January 2019, Accepted 14 February 2019) 


\section{Antibodly}

安迪浭㨋

\section{JiangSu LIBO Medicine Biotechnology Co.,Ltd.}

\section{Diagnosis of Hemolytic Disease of the Newborn}

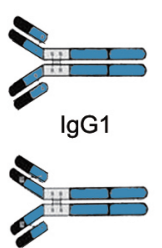

$\lg \mathrm{Ig}$

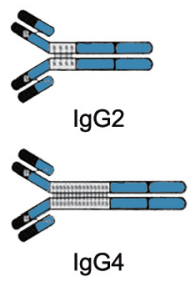

\section{IgG Subclasses Test Card}

The product is used for prediction of Hemolytic Disease of the Newborn, including the detection of Anti-A(B) IgG Subclasses of the Newborn Red Blood Cell and Pregnant Women Serum.

\section{Treatments for Hemolytic Diseases of the Newborn(Fetus) (For Reference Only)}

Pre-pregnancy — Effective Intervention

- Blood typing

- Detection of antibody potency

- Combination of traditional chinese and westem medicine treatment

- Plasmapheresis (Removal)

- High dose of gammaglobulin

\section{Pregnancy — Active Treatment}

- Monitor of antibody potency

- Combination of traditional chinese and westem medicine treatment

- Plasmapheresis (Removal)

- High dose of gammaglobulin

- Intrauterine transfusion

\section{Postpartum — Symptomatic Treatment}

- Hemolysis detection

- Phenobarbital

- Phototherapy

- Gammaglobulin and hormone

- Albumin

- Exchange transfusion in neonates
Early Treatment for Pregnant Women with High Potency

Traditional Chinese Medicine

- Herba Artemisiae Scopariae, Radix Astragali, Fructus Gardeniae

- Herba Taxilli, Semen Cuscutae, Radix Dipsaci Asperoidis

- Radix Angelicae Sinensis, Radix Paeoniae ALBA

- Rhizoma Atractylodis Macrocephalae

\section{Western Medicine}

- Vc: 100mg,1/d;

- VE: $100 \mathrm{mg}, 1 / \mathrm{d}$;

- Oxygen inhalation: 20min per time, 2/d; Plasma(Lymph) Exchange, Immunosorbent Intrauterine Transfusion

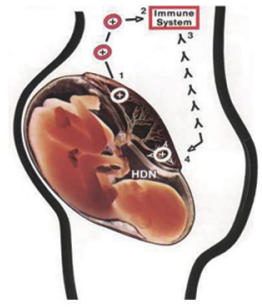

\section{Antibod 进迪溥据 \\ JiangSu LIBO Medicine Biotechnology Co.,Ltd.}

Address: No 78 West Dongsheng Road, Jiangyin, Jiangsu214400, P.R. China

Web Site: www.libiotech.com

Sales Tel: +86-510-86990618、+86-510-86990633

Tech Tel: +86-510-86990608、+86-510-86990655

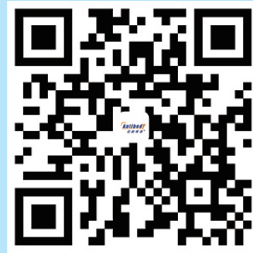

